TTR

Traduction, terminologie, re?daction

\title{
La traduction et le devenir social : le cas de l'irruption de la science-fiction américaine en France après la Seconde Guerre mondiale
}

\author{
Jean-Marc Gouanvic
}

Volume 7, numéro 1, 1er semestre 1994

Genres littéraires et traduction

URI : https://id.erudit.org/iderudit/037171ar

DOI : https://doi.org/10.7202/037171ar

Aller au sommaire du numéro

Éditeur(s)

Association canadienne de traductologie

ISSN

0835-8443 (imprimé)

1708-2188 (numérique)

Découvrir la revue

Citer cet article

Gouanvic, J.-M. (1994). La traduction et le devenir social : le cas de l'irruption de la science-fiction américaine en France après la Seconde Guerre mondiale. TTR, 7(1), 117-152. https://doi.org/10.7202/037171ar
Résumé de l'article

La traduction et le devenir social: le cas de l'irruption de la science-fiction américaine en France après la Seconde Guerre mondiale. À partir de la théorie de Pierre Bourdieu (concepts de champ, de capital et de biens symboliques, d'habitus et d'illusio), cette étude propose une sociologie de la traduction appliquée à l'importation de la science-fiction américaine en France dans les années 1950. C'est d'un « nouveau genre littéraire » d'origine américaine que Boris Vian, Raymond Queneau et Michel Pilotin se font les initiateurs dans l'espace socio-culturel français. Or, si les textes de SF des auteurs américains sont traduits massivement dans la culture française de l'époque, cette traduction n'a lieu que moyennant l'importation des structures institutionnelles américaines autonomes (en particulier des magazines et des collections spécialisées) qui ont émergées à la fin des années 1920 et à la naturalisation du modèle subculturel américain qui aboutissent à la constitution d'un champ de science-fiction autonome dans l'espace culturel français. Dès lors, la question traductologique essentielle que pose l'importation de la science-fiction américaine en France est la suivante. Lorsqu'un type de texte (ou un genre) prend corps dans un groupe social d'un espace culturel (source) et qu'il est traduit dans un autre espace culturel, par quel groupe social ce type de texte ou ce genre est-il reçu dans l'espace culturel cible? L'auteur fait l'hypothèse que la translation (au sens mathématique du terme) de la science-fiction américaine (textes et structures institutionnelles) réussit parce que, d'une part, il existe en France une ou des catégories sociales qui sont les homologues de la petite bourgeoisie américaine technophile des années 1920 et parce que, d'autre part, il existe une adhésion plus ou moins consciente à l'American way of life comme le modèle de société qui s'impose comme allant de soi dans de larges pans de l'espace social français de l'après-guerre. Dans ces conditions, la traduction contribue à renforcer le modèle américain dans sa prétention à l'universalité, quelles qu'aient été à l'origine les vertus de changement social que les agents d'implantation Vian, Queneau et Pilotin avaient reconnues dans la science-fiction et sur lesquelles ils s'appuyaient pour la présenter comme un « genre nouveau ».

Tous droits réservés ( TTR: traduction, terminologie, rédaction — Les auteurs, Ce document est protégé par la loi sur le droit d'auteur. L’utilisation des 1994 services d'Érudit (y compris la reproduction) est assujettie à sa politique d'utilisation que vous pouvez consulter en ligne.

https://apropos.erudit.org/fr/usagers/politique-dutilisation/ 


\title{
La traduction et le devenir social: le cas de l'irruption de la science- fiction américaine en France après la Seconde Guerre mondiale ${ }^{1}$
}

\author{
Jean-Marc Gouanvic
}

«Le monde est une branloire perenne.»
Montaigne

\section{Introduction}

Si, dès le début des années 1970, ce qu'on est aujourd'hui convenu d'appeler l' «école du polysystème» (représentée par les sémioticiens de Tel Aviv, Itamar Even-Zohar et Gideon Toury, et de Louvain, José Lambert) a entraîné une réévaluation des études théoriques en traduction en situant cette dernière dans le cadre général de la sémiotique des textes, du point de vue qui est ici le nôtre leur apport essentiel réside dans le fait que ces théoriciens ont été les premiers à conférer une place importante aux «normes traductionnelles ${ }^{2}$ » en vigueur dans les systèmes cibles à l'époque

1. Cette étude résulte d'une recherche subventionnée entre 1991 et 1993 par le Conseil de Recherche en Sciences Humaines du Canada et intitulée: «Discours sociaux et traduction: la traduction de la science-fiction américaine en France dans les années 1950).

2. Dans In Search of a Theory of Translation (Tel Aviv, The Porter Institute for Poetics and Semiotics, Tel Aviv University, 1980), Gideon Toury consacre plusieurs chapitres à la question des «Normes traductionnelles»: «Translated Literature, System, 
des traductions. Sur ces bases, il a été dès lors possible de construire la traduction en tant qu'objet théorique et historique, les deux instances ayant été jusqu'alors couramment dissociées pour déboucher soit sur une forme ou une autre de formalisme structuralisant, soit sur une conception purement historiographique de la traduction, la première étant entachée du vice de mettre sur le compte d'une prétendue "essence" des structures et des systèmes ce qui en réalité est déterminé par l'histoire de ces structures et de ces sytèmes; la seconde tombant dans le travers inverse qui consiste à s'arrêter à la simple description des événements, à placer sur un même plan des faits de poids historique très inégal.

Dans ce qui suit, il s'agira à partir de la traduction d'un genre littéraire senti comme d'apparition récente (la sciencefiction) d'une part de co-penser les "modèles génériques» et leur mode d'insertion dans la société et, d'autre part, de dégager la façon dont la traduction transforme et acclimate ces modèles d'origine étrangère. Un tel programme ne serait sans doute pas désavouć par les polysystémistes. Il nous semble cependant

Norm, Performance - Toward a TT-Oriented Approach to Literary Translation» (pp. 35-50); «The Nature and Role of Norms in Literary Translation» (pp. 51-62); «Equivalence and Non-Equivalence as a Function of Norms» (pp. 63-70); “Norms of Literary Translation into Hebrew" (pp. 122-139). Pour Toury, la traduction littéraire est soumise à des contraintes objectives (des règles quasi absolues) et à des idiosyncrasies totalement subjectives. Entre ces deux pôles extrêmes s'insèrent les facteurs intersubjectifs communément désignés sous le nom de "normes", lesquelles occupent plusieurs points dans le continuum des contraintes (p. 51). Sur ces bases, Toury distingue divers types de normes, les normes préliminaires et les normes opérationnelles; ces dernières sont dites textuelles ou matricielles (dans la mesure où elles se situent au niveau de la matrice du texte); elles sont textuelles, c'est-à-dire soit purement [sic] linguistiques (Toury place dans cette catégorie les normes stylistiques), soit littéraires, en fonction des genres, périodes, critères $d^{\prime}$ acceptabilité, techniques littéraires, etc. ( $p$. 54). 
difficile à réaliser par les seuls moyens fournis par ces derniers, car il y manque une composante théorique centrale: une sociologie des productions symboliques. Pour réaliser le programme que nous nous sommes tracé, nous ferons donc appel à la théorie de Pierre Bourdieu, laquelle nous paraît actuellement la plus apte à rendre compte des enjeux sociaux des productions symboliques. Comme Pierre Bourdieu n'a pas pensé sa théorie pour la traduction, cette étude pourra également être considérée comme une tentative pour adapter ses idées à la traduction. Il s'agit donc d'hypothèses de travail qui demanderont à être validées dans des travaux ultérieurs.

Ainsi, nous inspirant des travaux de Pierre Bourdieu ${ }^{3}$, nous pouvons dire que les textes étrangers traduits et mis sur le marché entrent dans la logique du marché des biens culturels. Dans l'économie libérale occidentale (avec des variations locales entre l'ultra-libéralisme et le libéralisme teinté de dirigisme d'État), c'est globalement la loi du marché, libre concurrence et «laisser-faire», qui régule la production, la diffusion et la consommation des biens culturels. L'éditeur soumis à la libre concurrence «cherche à imposer ${ }^{A} »$ dans le champ un nouveau

3. Les concepts théoriques élaborés par Pierre Bourdieu à partir du milieu des années 1960 . trouvent des formulations diverses de livre en livre et selon les objets d'étude. Dans le domaine des productions artistiques, on se référera minimalement aux ouvrages suivants: capital symbolique et marché des biens symboliques (1971, pp. 49-126), habitus (1979, pp. 189-195), champ (1992, pp. 321-326), illusio (1992, pp. 455-458).

4. À ceci près (et tel est le sens de nos guillemets) que l'investissement des agents dans la lutte pour occuper la meilleure position possible dans un champ s'opère non pas sur le mode de la stratégie délibérée, mais sur celui de l'ajustement au jeu qui se joue dans le champ (ce que Bourdieu nomme l'illusio, adhésion au jeu et sens du jeu) et qui, dans le même moment, institue le champ. Bourdieu l'exprime on ne peut plus clairement dans Leçon sur la leçon: "Le moteur - ce qu'on appelle parfois la motivation - n'est ni dans la fin matérielle ou symbolique de l'action, cornme le veut le finalisme naif, ni 
producteur et un nouveau produit vecteurs d'un nouveau système de goûts, et susceptibles d'entraîner un réagencement des hiérarchies de goûts en vigueur dans le champ. L'œuvre traduite diffusée dans le champ littéraire est soumise à la même logique objective que l'œuvre indigène non traduite: le monopole de l'imposition des catégories de perception et d'appréciation légitimes. La décision de traduire tel texte de tel auteur étranger est prise par un éditeur en fonction de l'anticipation du profit maximal qu'il estime pouvoir en retirer. La traduction d'auteurs et d'œuvres qui ont «fait leurs preuves» dans le champ culturel source (œuvres consacrées par des prix ou par des chiffres de vente qui en font des best-sellers) constituent en général des placements sûrs. Mais ce type de profit est le propre des éditeurs positionnés comme industrie culturelle. Les éditeurs n'assumant pas cette position justifient leur place dans le champ par la logique de la découverte. Il en résulte que l'origine étrangère de

dans les contraintes du champ, comme le veut la vision mécaniste. Il est dans la relation entre l'habitus et le champ qui fait que l'habitus contribue à déterminer ce qui le détermine." (1982, p. 48) Voir aussi dans Choses dites la critique de la notion de «stratégie»: "C'est encore un terme que je n'emploie pas sans hésitation. Il encourage le paralogisme fondamental, celui qui consiste à donner le modèle qui rend raison de la réalité pour constitutif de la réalité décrite, en oubliant le 'tout se passe comme si', qui définit le statut du discours théorique. [...] En fait, tout mon effort vise au contraire, avec la notion d'habitus, par exemple, à rendre compte du fait que les conduites (économiques ou autres) prennent la forme de séquences objectivement orientées par référence à une fin, sans être nécessairement le produit, ni d'une stratégie consciente, ni d'une détermination mécanique. Les agents tombent en quelque sorte sur la pratique qui est la leur plutôt qu'ils ne la choisissent dans un libre projet ou qu'ils n'y sont poussés par une contrainte mécanique. S'il en est ainsi, c'est que l'habitus, système de dispositions acquises dans la relation à un certain champ, devient efficient, opérant, lorsqu'il rencontre les conditions de son efficacité, c'est-à-dire des conditions identiques ou analogues à celles dont il est le produit." (1987, pp. 127-128) 
l'œuvre est négociée diversement pour l'usage du champ culturel cible. On peut dégager deux pôles: (A) L'origine étrangère de l'œuvre est proclamée de façon ostentatoire dans le cas où l'œuvre jouit d'un capital symbolique élevé dans la culture source ou encore dans le cas où la culture source elle-même est globalement empreinte d'une forte aura de légitimité. Cette légitimité peut pousser l'effet d'ostentation jusqu'à la traduction fictive ou fausse traduction. (B) A l'opposé, l'origine étrangère du texte peut être dissimulée de diverses façons: naturalisation du prénom et/ou du nom de l'auteur; remplacement du nom de l'auteur par celui du nom du traducteur, promu ainsi au rang d'autcur.

Sur ces bases méthodologiques, nous voulons rendre compte d'une dimension de la traduction, l'appartenance des textes traduits à des ensembles qui les subsument, les genres littéraires ${ }^{5}$, et qui leur confèrent leur existence socioinstitutionnelle et leur efficace discursive, en concentrant notre attention sur la science-fiction américaine traduite en français. Ce cadre théorique est particulièrement approprié à l'étude traductologique du genre science-fiction, du fait que ce dernier est marginalisé, son statut institutionnel étant celui du paralittérairc. La science-fiction fait partie de ces genres non canoniques, "dominés», qui, dans nos sociétés, sont maintenus pour des raisons idéologiques en dehors de la clôture lettrée (Angenot, 1980).

\section{La science-fiction: petite mise en place de poétique sociale}

Ce qui distingue la poétique de science-fiction de celle d'un récit réaliste, $d^{\prime}$ un récit fantastique ou encore d'un conte merveilleux, c'est la thématisation de ce que Darko Suvin appelle (en

5. Pour lever toute équivoque, soulignons que nous n'employons le concept de genres littéraires que dans le sens de configurations socio-esthétiques dotées d'une poétique, $d$ 'un telos et de potentialités discursives propres qui trouvent ou non à se réaliser à une époque et dans une société données. 
empruntant à Ernst Bloch) un novum. La science-fiction est ainsi définie par rapport aux autres genres littéraires:

SF is distinguished by the narrative dominance or hegemony of a fictional "novum» (novelty, innovation) validated by cognitive logic. (Suvin, 1979, p. 63)

Dans le même ouvrage, Suvin établit une autre distinction, cette fois entre le "vrai» et le «faux» novum, en reconnaissant le premier par ses «ethico-political liberating qualities» (ibid. p. 82).

Le théoricien de l'utopie et de la science-fiction par là ne reconnaît de pertinence qu'à la thématisation du socio-historique en science-fiction. Nous préférons nous référer au concept d'altérité, qui est plus englobant du fait qu'il prend en compte aussi bien la dimension bio-écologique que socio-historique de la thématisation. L'altérité bio-écologique s'exprime dans les récits de voyages spatiaux et de rencontres avec les entités extraterrestres. On peut penser aux romans - et aux films correspondants - Solaris, Rencontres $d u 3^{c}$ type, Alien, etc. Le plus remarquable est Solaris de Stanislas Lem, où l'altérité est globale: les cosmonautes découvrent une planète-océan métamorphosable dont les lois physico-chimiques sont radicalement différentes de celles sur lesquelles reposent les théories scientifiques admises dans l'univers terrestre référentiel. Pour saisir l'altérité de la planète-océan, les savants tentent par tâtonnements de construire une nouvelle science, la solaristique, et le récit se clôt sur le développement lent et incertain de cette science. Si Solaris est l'un des romans majeurs de la science-fiction moderne, c'est parce que la thématisation de l'altérité bio-écologique donne lieu à une réévaluation radicale des schèmes de pensée et des visions du monde, à un effet analogue à une rupture épistémologique.

Quant à l'altérité socio-historique, elle se trouve théâtralisée en particulier dans les utopies, les uchronies, les voyages dans le temps et les anticipations. Ce sont par exemple The Time Machine de H.G. Wells, un film comme Blade Runner (d'après le roman Do Androids Dream of Electric Sheep? de Philip Dick) ou le roman d'Ursula Le Guin The Dispossessed. Dans The Time Machine, par exemple, le voyage dans le temps est l'occasion 
d'une réflexion sur le devenir des sociétés humaines. Dans cette fable darwinienne, le voyageur du temps découvre que la bourgeoisie victorienne est destinée à être cannibalisée par le prolétariat et que le terme de l'ćvolution est la fin entropique et le retour au néant. Lorsqu'elle aborde les thématiques de l'altérité socio-historique, la science-fiction est principalement critique sociale et parfois exploration eschatologique, même si les «fins dernières" sont dans ce cas sécularisées. Quoi qu'il en soit, l'effet discursif demeure le même que dans les récits à caractère bioécologique: il s'agit de mettre en scène la rupture, de susciter la mutation, d'évoquer la métamorphose.

2. Bref historique de la constitution du genre «sciencefiction»

Les récits mentionnés plus haut et présentés comme typiques de la poétique de la science-fiction moderne appartiennent à une exception près (The Time Machine de Wells) au genre tel qu'il a trouvé à s'actualiser après la Seconde Guerre mondiale. Cependant la coupure historique a cu lieu avant la guerre.

\subsection{Le tournant des années 1920 aux États-Unis}

Il y a deux histoires de la science-fiction: celle qui précède la fin des années 1920 et celle qui suit cette période. Avant 1926, la science-fiction $n^{\prime}$ est pas reconnue comme genre littéraire. Les récits n'ont droit à l'existence qu'à la condition de rester diffus dans l'ensemble des productions culturelles. On ne peut donc pas encore parler de "science-fiction" stricto sensu, car cette désignation dénote l'homogénéité d'un champ et une existence unifiée qui n'aura lieu qu'après les années 1920. En réalité, la pléthore de termes pour cataloguer les textes qui en relèvent est un signe évident de cette dispersion et de cette dénégation: roman scientifique (Jules Verne), anticipation (H. G. Wells), fantaisie scientifique (H. G. Wells), roman d'hypothèse (Maurice Renard), roman fantastique (Jacques Spitz), même si ces auteurs écrivent bien de la science-fiction (leurs textes seront réédités sous l'étiquette «science-fiction» des décennies plus tard). Cette valse hésitation sur ce qui est en jeu dans ces récits est le signe 
d'une intense lutte de pouvoir pour l'imposition d'un type de texte dans le champ de cette littérature. Comme le remarque Pierre Bourdieu, «L'enjeu des luttes à propos du sens du monde social est le pouvoir sur les schèmes classificatoires et sur les systèmes de classement qui sont au principe des représentations et, par là, de la mobilisation et de la démobilisation des groupes [...].» (1979, p. 559) Dans le champ des littératures non mimétiques, la lutte des classements est manifestement tout aussi âpre que dans les autres champs du pouvoir.

\section{$2.2 \quad$ Les modèles fondateurs}

Que se passe-t-il aux États-Unis au début du $20^{\mathrm{e}}$ siècle et qui débouche sur la constitution de la science-fiction comme genre, quand rien de comparable n'émergeait dans la culture française à la même époque? Le tableau 1 est une récapitulation des modèles et des agents en présence dans les deux espaces socioculturels où se joue l'avenir du genre, modèles dont le poids socioesthétique a été déterminant dans sa constitution sous la forme qui s'est imposée comme dominante.

Dans la constitution de la science-fiction en tant que genre spécifique, trois noms ressortent, dont les œuvres s'ćchelonnent sur un siècle: Edgar Allan Poe (dont The Narrative of Arthur Gordon Pym date de 1837), Jules Verne (en 1863 est publié Cing semaines en ballon), traduit et publié en livre aux États-Unis dès 1873 , et H.G. Wells, dont The Time Machine paraît en $1895^{6}$.

$D^{\prime}$ autres types de récits exerceront une influence, par exemple ceux que l'on désignera plus tard du nom d'sheroic fantasy» et dont le paradigme apparaît en 1912 avec Under the Moons of Mars, d'Edgar Rice Burroughs. Aux États-Unis, comme en France, il existe des éditeurs spécialisés dans la littérature populaire et qui publient des magazines, dime novels et pulp-

6. Voir notamment au point 4.3 ci-dessous un commentaire sur la co-existence et le poids respectif de ces trois auteurs dans la constitution de la science-fiction. 
TABLEAU 1

Généalogie de la constitution de la science-fiction comme genre et de son importation-traduction en France (années 1950)

Les agents (auteurs, traducteurs, éditeurs)

\begin{tabular}{|l|l||}
\hline \hline \multicolumn{1}{|c|}{ ETATS-UNIS } & \multicolumn{1}{c|}{ FRANCE } \\
\hline 1837: Edgar Poe & 1852: Poe traduit par Baudelaire \\
\hline & $1863:$ Jules Verne \\
\hline 1873: Jules Verne traduit \\
(et publié en livre) & \\
\hline [G.-B.: 1887: H.G. Wells] & \\
\hline & [1895: Wells traduit au Mercure \\
de France]
\end{tabular}


magazines, où paraissent des récits qui mêlent aventure, science et technologie; ce sont notamment les Munsey magazines et des magazines d'ingénieurs Modern Electrics et Electrical Experimenter. Ces deux derniers sont d'une importance particulière, car $c^{\prime}$ est leur fondateur, Hugo Gernsback, qui aura l'idée en 1926 de créer Amazing Stories exclusivement consacré à la "scientifiction", étiquette supplantée par «science fiction» en $1929^{7}$. Entre temps, en 1915, était survenu un événement à l'origine de la vague de fond que connaîtront les magazines de science-fiction à partir d'Amazing Stories: la création du premier magazine spécialisé, Detective Story, qui ouvre l'ère de la spécialisation du marché des magazines.

\subsection{La mutation subculturelle: Amazing Stories (1926)}

Avec Amazing Stories, Gernsback est à l'origine du regroupement des conjectures romanesques rationnelles jusqu'alors dispersées, du mouvement d'autonomisation de ce champ et de l'émergence de la science-fiction comme type littéraire sui generis. Du point de vue de la traduction, cette émergence de la SF comme genre est fondamentale. La traduction prend ici la forme d'un parcours géopolitique transnational et «migratoire», réglé par les intérêts de groupes sociaux des sociétés source et cible. Dire qu'il s'agit d'un parcours, cela ne renvoie pas plus à un mouvement centripète qu'à un mouvement centrifuge; le trajet suivi par les textes et les agents est polycentrique, et leur circulation obéit à des déterminations qui ne relèvent exclusivement ni des sociétés source ni des sociétés cible. Dans le cas de la constitution du genre science-fiction, le parcours des agents (auteurs, traducteurs, éditeurs) est tel qu'il ne suffit pas de la penser en termes centrés sur la culture cible, comme on a trop eu tendance à le faire en traductologie. Ainsi, par exemple, Hugo Gernsback est un Luxembourgeois immigré aux États-Unis en 1905 à l'âge de 17 ans. Quant aux modèles d'Amazing Stories, ce sont, on l'a vu, Jules Verne, H.G. Wells, Edgar Poe. Or, l'un des grands modèles

7. C'est dans l'éditorial du numéro de juin 1929 de Science Wonder Stories (un de ses autres magazines de SF) que Hugo Gernsback emploie «Science Fiction» comme un syntagme autonome. 


\section{TABLEAU 2}

\section{Le texte de science-fiction et sa mise en marché}

\begin{tabular}{|c|c|}
\hline $\begin{array}{c}\text { PRO } \\
\text { (Le marché de grande } \\
\text { consommation) } \\
\text { [rémunéré] }\end{array}$ & $\begin{array}{c}\text { FAN } \\
\text { (Le ghetto subculturel: le fandom) } \\
\text { [non rémunéré] }\end{array}$ \\
\hline PRODUCTEUR & PRODUCTEUR \\
\hline $\begin{array}{l}\text { AUTEUR: Spécialisé en SF ou non } \\
\text { (pseudonymes et étiquettes } \\
\text { génériques) }\end{array}$ & $\begin{array}{l}\text { AUTEUR: Auteur ludique (avec ou } \\
\text { sans prétention littéraire) sinon se } \\
\text { fait récupérer par le circuit pro. (cf. } \\
\text { Serge Brussolo en France) }\end{array}$ \\
\hline $\begin{array}{l}\text { TRADUCTEUR: Auteurs maison } \\
\text { (cf. B.R. Bruss au Fleuve Noir) et } \\
\text { traducteurs professionnels (agences } \\
\text { et pigistes) }\end{array}$ & TRADUCTEUR: Quasi inexistant \\
\hline $\begin{array}{l}\text { ÉDITEUR: Collections spécialisées } \\
\text { (rarement non spécialisées) } \\
\text { Revues et magazines (aussi } \\
\text { prozines) }\end{array}$ & $\begin{array}{l}\text { ÉDITEUR: Surtout de périodiques } \\
\text {. fanzines (fictions, critiques et } \\
\text { études, illustrations) } \\
\text {. spécialisés ou généraux } \\
\text { (horrifiques, technologiques } \\
\text { [informatiques], érotiques, etc.) } \\
\text {. newszines (nouvelles et potins du } \\
\text { fandom) } \\
\text {. personalzines (centrés sur la } \\
\text { personne du «fanéditeur») }\end{array}$ \\
\hline DIFFUSEUR & DIFFUSEUR \\
\hline $\begin{array}{l}\text { Circuit des librairies générales et } \\
\text { spécialisées (office) } \\
\text { Revues: librairies et kiosques }\end{array}$ & $\begin{array}{l}\text { Circuit des librairies spécialisées } \\
\text { (consignation) et envois postaux } \\
\text { (abonnements) }\end{array}$ \\
\hline CONSOMMATEUR & CONSOMMATEUR \\
\hline $\begin{array}{l}\text { grand public } \\
\text { fans }\end{array}$ & $\begin{array}{l}\text {. boulimique et exclusif mais très } \\
\text { sćlectif (auteur, sous-genre) } \\
\text {. «collecting fan» } \\
\text {. clubs de fans }\end{array}$ \\
\hline $\begin{array}{l}\text { INSTANCES DE } \\
\text { CONSÉCRATION }\end{array}$ & $\begin{array}{l}\text { INSTANCES DE } \\
\text { CONSECRATION }\end{array}$ \\
\hline $\begin{array}{l}\text { Conventions (congrès): } \\
\text { inexistantes, mais le secteur } \\
\text { professionnel est toujours présent } \\
\text { dans les conventions de fans (pour } \\
\text { des raisons clairement } \\
\text { commerciales). }\end{array}$ & $\begin{array}{l}\text { Conventions: } \\
\text { - générales de science-fiction } \\
\text {. spécialisées (É.-U.: «Trekkies») } \\
\text { - prix des congressistes } \\
\text { - «happening» (plus/moins } \\
\text { ludique, plus/moins intello) } \\
\text { Prix: lecteurs (Hugo aux É.-U.; } \\
\text { Rosny Aîné en France) } \\
\text { Prix-jury (Nebula aux É.-U.) }\end{array}$ \\
\hline
\end{tabular}


de Verne est Poe: Verne a consacré un article à ce dernier et son Sphinx des glaces est une suite des Aventures d'Arthur Gordon Pym de Poe. Si derrière Verne il y a Poe, c'est un Poe revisité par Baudelaire et si le roman scientifique vernien lui-même est réinterprété selon les intérêts de la société américaine, l'agent de cette réinterprétation est un émigrant luxembourgeois éduqué en Europe. On voit combien il serait réducteur de faire de la sciencefiction une simple interprétation, transformation et appropriation américaine du modèle vernien.

Après la mutation subculturelle opérée par Amazing Stories, la science-fiction devient un phénomène «aussi endémiquement américain que le Western» (Ketterer, 1974, p. x). Edgar Poe jouit d'une position en apparence paradoxale dans la culture américaine. Considéré comme un écrivain mineur dans le champ de la culture américaine haute ${ }^{8}$, il est présenté comme l'un des modèles importants dans le champ de la science-fiction. Il est vraisemblable que cette situation est due à la position extrêmement favorable qui est la sienne dans la culture française grâce à Baudelaire et qui s'est transmise dans le roman scientifique par l'intermédiaire de Jules Verne.

\subsection{L'institution de la science-fiction}

Parler de genre littéraire à propos de la science-fiction, c'est dorénavant se référer à ce champ spécifique, à ses modèles socioinstitutionnels, à la subculture de science-fiction et à ses goûts, ses rites, ses jargons, ses comportements maniaques tout autant qu'à ses réalisations admirables. Le mode spécifique d'institutionnalisation (production, diffusion, consommation, consécration) du texte de science-fiction peut se schématiser comme dans le tableau 2.

8. Il existe de nombreux indices du statut marginal de Poe par rapport au canon de la littérature américaine. L'un des plus significatifs est son absence des panoramas littéraires du XIX siècle américain, par exemple, de l'étude classique de F. O. Matthiessen, American Renaissance: Art and Expression in the Age of Emerson and Whitman (New York, Oxford University Press, 1941). 
Deux remarques s'imposent sur la co-existence couramment vécue sur le mode conflictuel - des deux marchés. (1) La subculture de SF est le produit de la spécialisation professionnelle (et de l'autonomisation consécutive) du champ de la SF et non le produit d'une autogénération spontanée. (2) La subculture de SF singe largement la culture lettrée (cf. les prix). $\mathrm{Au}$ sein de la subculture (le fandom), la même lutte (que dans le circuit lettré) a lieu pour l'occupation hégémonique du champ de la SF.

\section{Le parachutage de la science-fiction américaine dans} l'espace culturel de la France de l'après-guerre

Le succès extraordinaire de la science-fiction aux États-Unis entraîne une redistribution complète des cartes de la traduction des textes. ${ }^{9}$. Cependant, cette redistribution ne s'opère pas immédiatement: les éditeurs, amateurs et traducteurs Régis Messac et Georges Hilaire Gallet essaient d'implanter la SF en France dans les années 1930 en créant revues, maisons d'édition, collections spécialisées, mais sans succès. Il faut attendre les années 1950 pour que le modèle importé des États-Unis s'implante durablement en France grâce notamment aux éditeurs, traducteurs et critiques Boris Vian, Raymond Queneau et Michel Pilotin.

\subsection{Les agents Vian, Pilotin et Queneau}

Immédiatement après la guerre, un groupe d'intellectuels en tête duquel se trouve Boris Vian découvre la science-fiction américaine $^{10}$. Vian avait parmi ses amis Michel Pilotin, amateur

9. Ce succès se mesure à l'explosion soudaine de l'institution "science-fiction" sous la forme de magazines et de collections spécialisés, à l'émergence des auteurs qui seront les classiques de l'après-guerre, notamment Asimov, Van Vogt, Sturgeon, Heinlein, Clement, Leiber.

10. Dans la revue Arts (numéro du 29 octobre-4 novembre 1953), R. Queneau public un article intitulé "La Science-fiction vaincra», où il fait état des activités science-fictives du groupe. Ils 
boulimique de science-fiction américaine. C'est ce même Pilotin qui allait publier plusieurs articles sur la SF américaine sous le pseudonyme de Stephen Spriel et diriger la co-édition de Gallimard avec Hachette au «Rayon Fantastique». Pendant des mois, selon le témoignage de Claude Léon, un ami du groupe, ils lisent les romans six par six, "dans la fièvre et l'enthousiasme», dit-il et la revue des "aficionados" Astounding Science Fiction" Parmi les romans, il y a The Martian Chronicles de Ray Bradbury, Pebble in the Sky d'Isaac Asimov, The World of Null-A d'Alfred Van Vogt, trois récits significatifs de la poétique de l'altérité qui distingue la SF américaine de la science-fiction pré-moderne ${ }^{12}$.

\section{1) Les textes traduits par Boris Vian}

Quelles sont les œuvres traduites par Vian et les stratégies traductionnelles et métatraductionnelles (les commentaires et les critiques) mises en place par Vian, Pilotin et Queneau pour faire reconnaitre la science-fiction américaine dans l'espace culturel français?

L'auteur de l'Herbe rouge et de l'Automne à Pékin est l'un des tout premiers traducteurs de la science-fiction américaine dès 1951. Il traduit d'abord «le Labyrinthe» de Frank M. Robinson,

fondent un club des Savanturiers (qui deviendra ensuite la Société d'Hyperthétique) dont font partie, entre autres, Gaston Bachelard, Jacques Audiberti, Pierre Kast, Michel Pilotin, Boris Vian, Raymond Queneau. Le club se réunissait au bar La Reliure pour y boire, écrit Queneau, des "galaxies, coquetelles d'une belle couleur bleue de l'invention de Boris Vian».

11. Propos rapportés par Noël Arnaud ("Les Vies parallèles de Boris Vian", Bizarre, n ${ }^{\circ} 39-40$, fév. 1966, p. 81). La revue Astounding Science Fiction marque un saut qualitatif par rapport à Amazing Stories: sous la houlette de John W. Campbell Jr. à partir de septembre 1937, Astounding publiera bon nombre des auteurs appréciés de Vian et de ses amis.

12. John $\mathrm{W}$. Campbell Jr. considère que la science-fiction de qualité commence seulement en 1945 avec The World of Null-A d'A.E. Van Vogt, que Vian offrira de traduire à Gallimard. 
pour les Temps modernes en 1951. Cette nouvelle accompagne le premier article de Vian et Pilotin sur la SF: «Un nouveau genre littéraire: la Science Fiction». Puis ce sont quatre traductionsadaptations publiées dans France-Dimanche en 1952 ${ }^{13}$ : «les Vivisculpteurs» de Wallace West, «le Veldt dans la nursery» de Ray Bradbury, "Pas bêtes, les gars de Bételgeuse» de William Tenn et «Si vous étiez un Moklin» de Murray Leinster. Trois de ces quatre nouvelles se caractérisent par leur ton humoristique, que Vian accentue nettement dans son adaptation. En $1953 c^{\prime}$ est la novella de Lewis Padgett «Tout smouales étaient les borogoves" dans le Mercure de France, la traduction de The World of Null-A pour «Le Rayon Fantastique» chez Hachette-Gallimard également en 1953 et sa suite The Pawns of Null-A en 1957 chez le même éditeur.

Voici un résumé des intrigues des quatre nouvelles publiées dans France-Dimanche, pour capter l'esprit de ce qui est en jeu dans les traductions effectuées par Vian.

- «Les Vivisculpteurs" est une histoire de résistance au vieillissement et de mise en échec de la mort. En 2400, les êtres humains sont capables de se régénérer, de filtrer leurs souvenirs, de réorienter leurs pulsions, ce qui a pour conséquence de rendre caduque l'idée qu'il existe une fatalité ou un destin.

- Dans «le Veldt dans la nursery", la nursery est une pièce à la technologie ultraperfectionnée où les enfants peuvent faire surgir par simple suggestion mentale le monde imaginaire de leur choix. Mais ce qui intéresse surtout les enfants, ce sont les animaux féroces du veldt africain. Craignant pour l'équilibre mental de leur progéniture, les parents décident de fermer la nursery. Ne l'entendant pas ainsi, les enfants attirent leurs parents dans la nursery après y avoir fait surgir le veldt et les lions, et ils les y. enferment, avec le résultat qu'on imagine.

13. Dans sa livraison du 6 janvier 1952 France-Dimanche titre pour annoncer la publication des quatre nouvelles américaines traduites par Vian: «Français, Attention: voici la sciencefiction!» 
- «Pas bêtes, les gars de Bételgeuse» est, sur le mode humoristique, une critique - ambiguë - du colonialisme par une sorte de mundus inversus. Des escargots extraterrestres à la politesse tout orientale débarquent un jour sur Terre. Aussi ridicules qu'elles puissent paraître, ces créatures sont dotées $\mathrm{d}^{\prime}$ une intelligence remarquable et d'une technologie avancée. Ainsi, elles possèdent le secret de la longévité: il suffit de se faire irradier tous les dix ans dans des revitaliseurs. Intéressés, les Terriens acceptent de vendre les éléments radioactifs purs de la Terre contre des revitaliseurs. Les escargots, satisfaits du marché, repartent d'où ils étaient venus. La Terre connaît une ère de bonheur, jusqu'au moment où les revitaliseurs tombent en panne, sans qu'il soit possible de les réparer, car on s'aperçoit qu'ils tirent leur énergie de la radioactivité. Il y a deux morales à cette histoire. L'une est politique et pragmatique (elle promeut les seuls intérêts nationaux): «Rappelez-vous le chef indien qui vendit New-York pour deux caisses d'eau de feu». La deuxième est idéaliste et euphorisante: devant l'adversité les nations de la Terre $s$ 'unissent, forment un gouvernement mondial et parviennent à fabriquer de la radioactivité artificielle.

- «Si vous étiez un Moklin» se passe dans un comptoir commercial sur une planète lointaine, où les indigènes très pacifiques possèdent le pouvoir étrange d'imiter les Terriens par sympathie. Pour leur rendre hommage, les Moklins offrent aux humains leurs répliques exactes en de nombreux exemplaires. Ce cadeau en apparence flatteur est empoisonné: les répliques sont dotées des caractères dont les humains sont les plus fiers. Pour conserver leur supériorité sur les répliques, les Terriens n'ont d'autre solution que de quitter la planète.

\section{2) Les stratégies d'implantation et de consécration}

Pourquoi Vian choisit-il de traduire ces textes? Ils possèdent des caractères qui sont en apparence contradictoires: ce sont des récits légers et des critiques sociales acérées, que Vian a manifestement pris plaisir à traduire. Ainsi, Pierre Kast rappelle "quel plaisir extrême Boris Vian avait pris à traduire la plus violente des nouvelles de Ray Bradbury «le Veldt dans la 
nursery", consacrée avec une vengeresse allégresse au prétendu paradis de $l^{\prime}$ enfance ${ }^{14} »$.

Ces traductions sont justifiées non par des nécessités économiques mais par des stratégies de diffusion, de légitimation et de consécration du genre. Et dans ces stratégies entrent des pratiques autres que spécifiquement traductionnelles. C'est le cas de l'article des Temps modernes, suivi de la traduction de la nouvelle de Frank M. Robinson. Cette nouvelle est une sorte d'illustration de l'essai de Vian-Spriel. La mise en relation étroite de la fiction avec le discours critique offre un modèle de lecture de l'une par l'autre. Or, l'analyse comparative des discours fictionnels en traduction et des discours critiques fait apparaître une remarquable cohérence des topoï.

\section{a) Le topos du «genre nouveau»}

Les articles non seulement de Vian-Spriel, mais de Spriel et de Queneau, proclament que la science-fiction est un genre «nouveau»: c'est l'attaque préférée du trio, la démonstration consistant à dire en quoi il est nouveau. Ce qui le rend "nouveau", c'est d'abord qu'il opère la synthèse des deux cultures, la scientifique et la littéraire. Pour Vian, le lecteur idéal de science-fiction,

$c^{\prime}$ est le mathématicien, le physicien ou les gens très cultivés du modèle de Raymond Queneau, qui savent à la fois ce que l'on fait en littérature, ce que l'on fait en mathématiques, ce que l'on fait en physique. Ce sont les gens qui ne font pas un mur entre eux et une partie de la connaissance ${ }^{15}$.

La science-fiction américaine met sous les yeux de l'homme moderne une image non spécialisée de lui-même que Vian n'hésite pas à rapprocher de celle de l'homme de la Renaissance.

14. Pierre Kast, «Boris Vian», préface à la réédition de l'Herbe rouge, l'Arrache-cceur, les Lurettes fourrées (Paris, Pauvert, 1962), p. 20.

15. "Pierre Kast et Boris Vian s'entretiennent de la Science-fiction», $l^{\prime} E ́ c r a n\left(n^{\circ} 1\right.$, janvier 1958). 
Vian, dans «Un robot-poète ne nous fait pas peur», écrit: "[On veut] faire de nous des travailleurs spécialisés [...]. Refusons [...]. Sachons tout. [...] L'avenir est à Pic de la Mirandole. ${ }^{16}{ }_{\text {» }}$

\section{b) Le topos de la contestation des valeurs traditionnelles}

Le second topos en ce qui touche la traduction de la sciencefiction américaine est la contestation des valeurs traditionnelles. Nous avons déjà mentionné le plaisir pris par Vian à traduire «The Veldt» de Bradbury. C'est aussi pour son pouvoir rupturel que Vian choisit de traduire The World of Null-A. Ce roman est fondé sur les théories largement contestées d'Alfred Korzybski ${ }^{17}$. Pour Korzybski, d'une part notre langage reposerait sur le principe aristotélicien d'identité et de non-contradiction, d'autre part ce langage ne serait doté d'aucune nécessité absolue comme le montrerait (selon Korzybski) l'étude du langage mathématique. Pour l'auteur, une grande partic des maux dont souffre l'humanité proviendrait de l'inadéquation de notre langage avec les choses. Une telle théorie a l'avantage d'être grosse de potentialités pour l'écrivain de science-fiction, et les deux romans de Van Vogt traduits par Vian réussissent à merveille à évoquer cette société d'êtres humains qui parviennent sur Vénus à vivre sclon une logique non-aristotélicienne au terme d'une discipline

16. «Un robot-poète ne nous fait pas peur», Arts (10-16 avril 1953). Repris dans le recueil Cantilènes en gelée (Paris, U.G.E., coll. "10/18", 1970), p. 225.

17. La Sémantique générale d'Alfred Korzybski n'est pas sans relation avec ce que l'on nomme communément $l^{\prime}$ ' Hypothèse Sapir/Whorf», selon laquelle «le langage est avant tout une classification et une réorganisation opérées sur le flux ininterrompu de l'expérience sensible, classification et réorganisation qui ont pour résultat une ordonnance particulière du monde» (B. L. Whorf, cité par Georges Mounin, Problemes théoriques de la traduction, Paris, Gallimard, 1963, p. 46). Cette conception, parfois désignée sous le nom d'hypothèse «de Sapir, Korzybski et Whorf» (Mounin, ibid. p. 46, n. 2), est critiquée par Roman Jakobson dans «On Linguistic Aspects of Translation" (On Translation, Reuben A. Brower, dir. Oxford, Oxford University Press, 1966 [1 ${ }^{\text {ère }}$ éd.: 1959], pp. 232-239). 
toute mentale. La nouvelle de Lewis Padgett «Tout smouales étaient les borogoves" (dont le titre est une citation de Through the looking-glass de Lewis Carroll) entretient aussi avec les théories de Korzybski une relation privilégiée. Cette fois-ci, la Sémantique générale sert à redéfinir la démence et la normalité selon des critères non-aristotéliciens. Ces œuvres de Van Vogt et de L. Padgett sont des exemples de la rupture épistémologique qui caractérise la SF américaine. Elles constituent une remise en question radicale des valeurs selon des paradigmes fictionnels non mimétiques.

\section{c) L'«ivresse de la raison»}

Enfin, Vian choisit la SF américaine pour la façon qu'elle a $\mathrm{d}$ 'induire la lucidité du lecteur tout en gardant intact le plaisir de lecture: «La science-fiction double l'excitation affective d'une sorte d'ivresse de la raison de goût très spécial. ${ }^{18}$ "Vian ne se fait pas l'apologiste d'une SF cérébrale: il craint l'esprit de sérieux par dessus tout et se garde bien de faire sortir la SF du circuit populaire.

3.2. Les points de chute de la science-fiction: la naturalisation des structures éditoriales américaines

La traduction de la science-fiction américaine en tant que genre, c'est avant tout l'importation de structures institutionnelles (notamment éditoriales) homologues à celles qui existent dans la culture source. Sans ces structures, la traduction des textes $n^{\prime} a$ pas lieu. Or, ces structures institutionnelles ne sont pas innocentes: elles sont porteuses de modèles qui déterminent des positions sur le marché des biens symboliques. Le modèle gernsbackien de constitution du genre SF est celui qui a réussi, mais cette réussite a été chèrement payée par une marginalisation et une ghettoisation de cette littérature d'une part et par une coupure par rapport à la tradition de l'utopie et du débat social d'autre part. La SF du ghetto s'est complètement coupée des courants

18. Boris Vian et Stéphane [sic] Spriel, "Un nouveau genre littéraire: la Science-fiction", les Temps Modernes ( $n^{0} 72$, octobre 1951). 
utopiques, pourtant bien vivants avant elle, alors qu'elle faisait une place au bizarre, à l'étrange et à certaines formes du fantastique, comme la fantasy ${ }^{19}$. La fantasy et le fantastique, avec leurs modèles discursifs propres, ont toujours tendu à entrer en polémique avec la SF en flattant les tendances antiscientifiques et ésotériques d'une partie du lectorat de SF.

Cela dit, voici quelques repères historiques, où apparaissent la manière française de naturaliser les structures institutionnelles d'origine américaine.

1950: Pour la première fois l'expression «science fiction» apparaît en français sur la jaquette de la traduction du roman The Humanoids de Jack Williamson publiée chez Stock. Ce sera le seul titre de la collection.

1951: Les éditeurs Hachette et Gallimard s'allient pour publier la collection "Le Rayon Fantastique», où sont traduits les grands classiques de la science-fiction américaine (et anglaise, dans la foulée): en tout 61 traductions entre 1951 et 1958.

1951: Les éditions Fleuve Noir fondent la collection «Anticipation», qui publiera 150 titres entre 1951 et 1960 . La place qui y est faite aux traductions est réduite: sur ces 150 titres, une trentaine de traductions-adaptations dont la plupart d'un auteur britannique sans influence sur le genre ${ }^{20}$.

1954: Les éditions Denoël prennent position dans le champ de la science-fiction avec la collection «Présence du futur».

19. Il n'est pas indifférent pour les traductologues que la fantasy soit sentie comme appartenant spécifiquement à la culture anglo-américaine, au point où aucune traduction française n'existe encore pour ce terme, même si d'assez nombreuses auteures francophones écrivent des récits de ce type. Tout se passe comme si le raccrochage (par la graphie) à un modèle anglo-américain constituait une plus-value dans le champ et devait, à ce titre, être préservée. On a ici un effet tout à fait analogue à celui de l'importation de la Science Fiction, quoique à une moindre échelle.

20. Il s'agit de John Russell Fearn (1908-1960), connu des lecteurs de la collection «Anticipation» sous les pseudonymes de Vargo Statten, Volsted Gridban et Vector Magroon. 
1953: Fondation de la revue Fiction, par Maurice Renault, publicitaire passionné de littérature policière. $C^{\prime}$ est pour publier une revuc de littérature policière (Mystère-Magazine en 1948) qu'il avait fondé les éditions OPTA. Mystère-Magazine, l'édition française de l'Ellery Queen's Magazine, connaîtra un succès foudroyant. Renault se laisse convaincre de publier une adaptation française du Magazine of Fantasy and Science Fiction, $\mathrm{d}^{\prime}$ Ellery Queen ${ }^{21}$, même si Renault ne s'intéresse pas du tout à la science-fiction.

1953: Galaxie ( ${ }^{1}$ èr série) paraît aux éditions Nuit et Jour, éditeur du magazine populaire Radar alors disparu. C'est l'édition française de Galaxy Science Fiction dont elle reprend le logo américain tel quel. Ici tout est traduit du magazine américain, mais avec beaucoup de désinvolture: les textes sont mutilés, raccourcis pour des raisons d'espace. Paradoxalement, ce travail de traduction peu soigné et tronqué est intéressant en ce qu'il correspond souvent à un effort pour vraiment adapter le texte à la culture cible. Le nom des héros est francisé et parfois même celui des auteurs. Il reste que, vice rédhibitoire, la lecture est gênée par l'absence de contrôle de la qualité. De nombreuses nouvelles et huit romans américains ont été publiés entre 1953 et 1959, date de fin de la première série de Galaxie (France). La quasi-totalité des textes de cette première série sera rééditée entre 1973 et 1977 après retraduction complète par la revue Marginal. Une deuxième série de Galaxie sera lancée en 1964 sur des bases éditoriales sérieuses proches de celle de Fiction.

De 1958 à 1963, paraîtra la revue Satellite. Elle éditera de nombreuses nouvelles et une quinzaine de romans traduits de l'américain, sans attaches éditoriales américaines (contrairement à Fiction et Galaxie).

\section{Les modèles socio-discursifs en présence}

Par «modèles socio-discursifs», nous entendons les classes de récits (hérités ou non des traditions nationales indigènes) qui, à travers des thématiques relativement homogènes, actualisent des

21. Pseudonyme collectif de Frederic Dannay et Manfred B. Lee, tous deux américains. 
positions idéologiques dans l'espace social à une époque donnée. Ainsi, dans le champ des littératures populaires de l'Angleterre des années 1870 , font florès des récits de guerres futures. Ces récits visent à galvaniser les forces nationales pour la préparation de la prochaine guerre et pour la victoire grâce aux innovations technologiques. Le prototype en est The Battle of Dorking (1871), qui sera traduit en de nombreuses langues et connaîtra de multiples imitations. Ce modèle du récit des guerres futures tombera en désuétude à partir des années 1890 avec l'agglomération de thèmes hétérogènes au discours sur la défense de la nation, en particulier des intrigues sentimentales. Pour ce qui est de la France des années 1950, il existe un certain nombre de modèles disponibles, indigènes ou étrangers, et nous allons maintenant examiner quels sont ceux que les agents d'importation de la SF américaine en France ont cherché à acclimater. Procédons chronologiquement, en commençant par les livres.

\subsection{Les livres publiés dans les collections spécialisées nouvellement créées}

"Le Rayon Fantastique» chez Hachette-Gallimard en dépit de son nom n'a absolument rien à voir avec le fantastique: $95 \%$ des récits sont de la science-fiction pure et dure, avec une dimension vraiment scientifique, conformément aux idées de Raymond Queneau et de Boris Vian sur la SF. Cette collection ne compte pas que des chefs-d'œuvre, mais l'ensemble demeure de très haute qualité (les éditions J'ai lu rééditeront presque tous ces titres dans les années 1970, ce qui est la preuve de la qualité de cette collection). Pourquoi Fantastique, peut-on se demander? Gallimard, qui appartient au circuit lettré, n'est pas d'emblée prêt à lier son nom à cette littérature qui soulève les passions, la science-fiction, en dépit de la caution de Raymond Queneau. En outre, on se souviendra que Gallimard avait immédiatement avant la guerre publié les romans de science-fiction de Jacques Spitz sous l'étiquette "romans fantastiques». Au-delà de Spitz, Fantastique renvoie bien sûr à Mérimée et à Maupassant. La désignation de fantastique est donc ici sentie comme une contrainte par laquelle il faut passer pour publier de la sciencefiction. Cette contrainte est encore extrêmement forte dans le champ littéraire français après la guerre, comme l'atteste le titre 138 
d'un des premiers livres publiés sur la science-fiction en 1958: Une succursale $d u$ fantastique nommée science-fiction, de Jacques Sternberg ${ }^{22}$. L'auteur de cet essai dilue la science-fiction dans le grand ensemble du fantastique en ne lui reconnaissant pas de spécificité; il est à noter que cette position est largement responsable de l'état de dispersion dans lequel ont été maintenus les romans de conjecture rationnelle avant Gernsback.

En 1951 également apparaît la collection «Anticipation» des éditions Fleuve Noir. Le nom de la collection renvoie à la tradition des romans populaires et non à la littérature lettrée comme le fantastique. Le représentant par excellence de l'anticipation est en France Albert Robida; son œuvre a été publiée dans le circuit de la littérature populaire notamment sous forme de fascicules. Le titre "Anticipation» ne correspond guère aux thèmes abordés dans les livres qui sont surtout des aventures spatiales et extraterrestres sans aucun effort pour anticiper sur un état à venir de la société ou même sur la conquête de l'espace ${ }^{23}$. Cette collection "de hall de gare" (comme la nomment ses détracteurs) est le visage honteux de la science-fiction, au point que la revue Fiction l'occultera pendant des décennies, ne lui accordant pas une ligne de compte rendu critique.

«Présence du futur» des éditions Denoël est la collection intellectuelle et lettrée, celle qui profite du mouvement d'intérêt pour la SF tout en maintenant une distance respectable avec les auteurs que publie par exemple «Le Rayon Fantastique». On ne s'autorise de la SF chez Denoël que les auteurs poétiques et nostalgiques comme Ray Bradbury, qui est le premier auteur de la collection, les auteurs fantastiques comme Lovecraft (c'est la collection où le fantastique lettré est le plus présent) et les auteurs du mainstream (c'est-à-dire du champ de la littérature "générale», non-SF) français comme Jean-Louis Curtis, lesquels sont dans la tradition de la conjecture rationnelle française

22. Paris, Le Terrain Vague, 1958.

23. Voir au point 4.2 l'usage fait par la revue Fiction du terme «Anticipation». 
d'avant la guerre. Ici, le titre connote faussement des récits anticipatifs, que la collection public peu.

\subsection{Les périodiques: la revue Fiction}

Le paratexte de la revue Fiction est extrêmement révélateur de la position que la revue entend occuper dans le champ de la science-fiction. Son sous-titre est: "La revue de tous ceux qui s'intéressent à la fiction romanesque dans le domaine de l'étrange, du fantastique, du surnaturel, de l'anticipation scientifique». Fiction d'emblée se situe dans la tradition française de mélange des genres non mimétiques, cette tradition même qui - encore une fois - a contribué à maintenir les conjectures romanesques rationnelles dans un état complet de dispersion autant aux États-Unis qu'en France. Fiction publie un peu de tout, notamment des auteurs français du circuit lettré, tel qu'André Maurois, et les textes de science-fiction américains modernes du Magazine of Fantasy and Science Fiction en traduction présentés sous l'étiquette de l'anticipation scientifique. Cette étiquette n'est pas indifférente: l'anticipation est la désignation générique dominante dans les conjectures rationnelles de tradition française. Elle fait l'objet d'un consensus très large dans l'espace social français, par la convergence de l'anticipation populaire à la Robida, des anticipations wellsiennes fort appréciées des lettrés ${ }^{24}$ et même par une lecture anticipative de Jules Verne, promu au rang de prophète du développement scientifique. Cette désignation est extrêmement opératoire dans la mesure où elle est fondée sur un topos hégémonique de la théâtralisation de l'histoire dans la littérature: il existe des lois de l'histoire qui ne sont pas différentes des lois de la nature et on peut prévoir l'avenir comme on peut prévoir les réactions des corps physiques et chimiques, les mêmes causes produisant les mêmes effets. Cette conception positiviste de l'histoire est aussi celle de la science-fiction à l'américaine, à la différence près que les auteurs américains sont fascinés par les développements des merveilles

24. Cf. l'étude que nous avons consacrée à la réception de Wells dans la NRF: «H.G. Wells et sa réception dans la Nouvelle Revue Française (fév. 1909 - août 1914)», Conseil International des Études Francophones, Tucson, Arizona, 1991. 
scientifiques et technologiques et de leurs effets sociaux, quand les auteurs français ressentent une peur panique à l'égard de la science, ne la mettant en scène que pour en faire voir le visage déshumanisant et pour présenter l'histoire comme un enfer et la société future comme une fourmilière dans laquelle la liberté individuelle a disparu. Les modèles de cette idéologie hégémonique, ce sont Aldous Huxley (Brave New World) et George Orwell (1984), qui appartiennent au circuit lettré et refusent farouchement tout lien avec la science-fiction. Le refus militant de se trouver mêlé à la SF est l'indice d'un positionnement idéologique exclusif dans le champ de la littérature lettrée. Fiction peut assumer une position privilégiée de rassemblement de tendances traditionnellement antagonistes dans le champ des littératures non mimétiques, par une convergence exceptionnelle d'une part du Fantastique canonique à la française, de celui d'Edgar Poe revisité par Baudelaire et de la sciencefiction américaine. Seule la collection "Anticipation" des éditions Fleuve Noir est exclue de ce que Fiction estime acceptable dans le champ nouvellement constitué.

\subsection{L'hétérogénéité des modèles discursifs: le ménage à trois Verne-Wells-Poe}

L'analyse des modèles discursifs dans le genre SF permet de délimiter dans l'ensemble des textes publiés dans la culture française les modèles traductionnels qui trouvent à s'actualiser. Si la SF américaine, on l'a vu, s'est constituée sur le modèle prédominant du roman scientifique vernien, mais également sur le conte étrange à la Poe et sur le modèle du roman de critique sociale d'H.G. Wells, ces trois modèles sont loin de faire bon ménage. Le ménage à trois est possible à condition que la suprématic de Verne soit incontestée, Wells jouant les seconds violons et Poe ne recevant qu'une portion congrue de l'espace d'édition. Il demeure que la niche faite au conte étrange ou bizarre ("weird tale») dès la constitution du genre est un précédent dont les imitateurs pourront se réclamer sans risquer d'être accusés de trahir le modèle fondateur. 
4.4. Modèles discursifs antagonistes et choix de textes à (faire) traduire

L'intérêt d'une étude des modèles traductifs d'un genre nous paraît riche d'enseignements pragmatiques. Ainsi, l'auteur qui écrit de la SF, l'éditeur qui en publie, le traducteur qui en traduit bon gré mal gré situent leur activité par rapport aux positions possibles dans la tradition du genre telle qu'elle trouve à se réaliser dans le champ à l'époque en cause (contribuant ainsi à modeler le champ). L'éditeur français peut souhaiter faire traduire les récits et les auteurs américains qui sont les vecteurs de modèles socio-discursifs en vogue aux États-Unis. Cet éditeur français a devant lui une gamme de textes appartenant à des modèles socio-esthétiques extrêmement différents: (1) l'«heroic fantasy» à la Conan (de Howard), modèle des épopées fantastiques barbares, mélanges paranoïaques de force brutale et d'ésotérisme mystique ${ }^{25}$; (2) l'anti-utopie (ou dystopie) à la Huxley sur le thème notamment des dangers de la science et les risques de déshumanisation; (3) l'utopie annonçant des lendemains qui chantent (État mondial, village planétaire, écologisme...); (4) les fictions psychologisantes: si la SF s'est constituce sur l'appel de la nouvelle frontière, celle de la conquête de l'espace cosmique, aujourd'hui le modèle dominant est celui qui prétend découvrir le seul espace qui compterait: l'espace intérieur... Qu'il en ait conscience ou non, l'éditeur prend position sur la question de l'avenir social en choisissant ce qu'il fera traduire.

\subsection{Transformation et détournement discursifs}

Les différents textes sont extrêmement lourds de modèles idéologiques antagonistes, et le choix de l'éditeur est déterminant lorsqu'il s'agit de décider de ce qui va être traduit. Il en découle

25. C'est ainsi que, dans les années 1980 et faisant suite au succès de films tels qu'Excalibur, la plupart des éditeurs français (sauf quelques-uns comme Robert Laffont) se sont engouffrés dans la vogue de l'aheroic fantasy" américaine. Ce type de récits a, après quelques années, retrouvé sa médiocre place dans le champ de la science-fiction française. 
que les déplacements, manipulations, transformations que peut opérer une traduction sont souvent quantité négligeable dans la signifiance générale du texte traduit ${ }^{26}$. Il en va tout autrement lorsqu'il s'agit d'une adaptation, mais dans ce cas les déplacements sont ceux que l'on constate aussi dans la traduction intersémiotique (pour parler comme Jakobson) qui n'est pas une traduction stricto sensu. S'il y a transformation discursive, c'est beaucoup plus un effet de réception dans la culture cible par un détournement - très fréquemment observé par les traductologues - de l'œuvre originale. Alors, les modèles socio-esthétiques sont mis dans la société cible au service d'intérêts idéologiques sans relation avec ceux du texte dans la société source. C'est ainsi qu'un auteur américain comme Norman Spinrad a été adopté en France dans les années 70 par l'extrême-gauche post-soixantehuitarde $\mathrm{c}^{27}$. Philip K. Dick a subi un autre type de manipulation.

26. C'est ce que nous constatons dans notre étude: «Sémiotique et traduction: les enjeux sociaux de la traduction de la sciencefiction américaine au Rayon Fantastique (Hachette-Gallimard)", Actes (sous la direction de Ginette Adamson et Jean-Marc Gouanvic) du congrès mondial du Conseil International des Études Francophones tenu à Casablanca en juillet 1993. A paraître en 1995 en co-édition Hurtubise-HMH (Montréal) et Eddif (Casablanca).

27. Bernard Blanc écrit: “D'autres, comme Norman Spinrad, font le même boulot lque l'auteur français Jean-Pierre Andrevon et que le magazine La Gueule ouverte] outre-Atlantique. Dans Jack Barron et l'éternité (Laffont) il montre comment la science sert les intérêts d'un milliardaire au détriment du peuple. A travers cette analyse sans fard de l'Amérique contemporaine perdue dans ses rêves mégalomanes l'auteur démonte une machination hautement symbolique. [...] Avec un langage volontairement ordurier (car, après les Black Panthers, Spinrad a compris que dans certains cas l'injure peut avoir puissance révolutionnaire) Jack Barron et l'éternité montre le vrai visage de la science dans notre société: moyen, littéralement, de sucer la substance des peuples au profit des Dracula au pouvoir.» («Science-fiction et écologie», Univers 8, 1977, pp. 178-179) L'œuvre étrangère traduite est clairement mise au service des intérêts du groupe d'extrême-gauche de la science-fiction française; cependant le langage injurieux de l'auteur de science-fiction ne va pas de soi, 
Alors qu'aux États-Unis elle ne trouvait qu'un écho assez mitigé (sauf auprès de l'équipe de Science-Fiction Studies), en France elle a connu un succès considérable ${ }^{28}$ jusqu'à être récupérée après coup par Jean Baudrillard. C'est un autre cas patent d'appropriation de modèles socio-esthétiques par l'opération de traduction. Comment s'effectue cette manipulation? Chaque cas est à examiner soigneusement. On pourra se reporter à l'étude de sociosémiotique comparée que nous avons publiée ailleurs ${ }^{29}$ et qui montre que, contrairement à ce que Clem Robyns a trouvé en ce qui concerne la traduction de la Série noire ${ }^{30}$, la traduction de la science-fiction $\mathrm{n}^{\prime} \mathrm{a}$ - en dépit de sa marginalité et de son éviction hors du cadre de la Littérature - été qu'extrêmement rarement prise à la légère et à notre connaissance jamais été

puisqu'il faut convoquer les Black Panthers, dont la légitimité révolutionnaire semble inattaquable aux yeux du groupe, pour justifier ce langage «volontairement ordurier».

28. Philip K. Dick fut l'invité du Festival de science-fiction de Metz en 1977, dans le cadre duquel il prononça une conférence que Jacques Goimard a publiée dans l'Année 1977-1978 de la sciencefiction et du fantastique (Paris, Julliard, 1978). Dans sa présentation, J. Goimard écrit: «De tous les auteurs de sciencefiction américains, Philip Dick est certainement le plus populaire en France: des romans comme le Maitre du haut château et Docteur Bloodmoney (J'ai Lu) ont comblé l'attente d'une génération de lecteurs." (p. 127) Côté américain, Carl Freedman écrit dans Science-Fiction Studies: "When Philip K. Dick died in 1982, his career could not have been reckoned as precisely a failure, but neither was it, by the usual criteria, a roaring success." («Editorial Introduction: Philip K. Dick and Criticism", $n^{\circ} 45$, juillet 1988 , numéro spécial consacré à $P$. K. Dick. Repris dans On Philip K. Dick: Forty Articles from ScienceFiction Studies, Terre-Haute, Indiana, SF-TH Inc., 1992, p. 145). Roger Bozzetto, quant à lui, parle d'une "love story" entre Dick et la France («Dick in France: A Love Story», ibid., p. 153).

29. Voir note 26 .

30. Clem Robyns, "The Normative Model of Twentieth Century Belles Infideles: Detective Novels in French Translation", Target (vol. II, nº 1, 1990, pp. 23-42). 
traitée sur le mode des Belles Infidèles ${ }^{31}$. Le rapport de la France de l'après-guerre avec les États-Unis semble sans commune mesure avec le rapport qu'entretient la culture française classique avec l'Antiquité gréco-latine.

\section{L'implantation durable de la science-fiction (américaine) en France}

Les activités traductionnelles (de Vian) et métatraductionnelles des Vian, Queneau et Pilotin entraînent une mutation brutale et profonde du paysage culturel et institutionnel du secteur traditionnellement occupé par la littérature enfantine (et notamment par Jules Verne et ses avatars). Ces agents sont au cours des premières années à peu près les seuls à occuper le champ traductionnel aussi bien que métatraductionnel: ils mettent en place des stratégies d'intervention qui font preuve d'un sens aigu des enjeux discursifs et des conditions de légitimité. Le choix des récits à traduirc, les points de chute institutionnels tant de ces traductions que du discours critique (les Nouvelles littéraires, les Temps modernes, Critique et l'Observateur) imposent la science-fiction moderne de façon irréversible, même si des opposants farouches expriment très tôt leur aversion pour le genre. Ce sont par exemple les prises de position des Michel Butor (qui parle de "crise de croissance de la science-fiction» en 1953), Arthur Koestler ("Lorsque l'ennui naît de la fantaisie», 1953), Suzanne Breuil («la Science-fiction ou escroquerie à la science?» dans les Lettres françaises en 1955), Charles Dobzynski («Une machine à décerveler» également dans les Lettres françaises, 1956), René Marill Albérès («Faillite de la fiction scientifique», 1957), François Truffaut (« $\AA$ bas la science-

31. Les traductions-adaptations tronquées, non signées, publiées dans Galaxie ne peuvent en aucune manière être assimilées aux Belles Infidèles. Bien au contraire, elles sont un exemple exceptionnel dans le champ de la science-fiction - de cynisme mercantile qui tente de tirer profit du statut paralittéraire et subculturel du genre. Cette tentative échouera (un Galaxie $2^{\mathrm{e}}$ série paraît en 1959) sous la pression des autres agents du champ de la SF qui prennent le genre au sérieux et le considèrent comme de la littérature à part entière. 
fiction", 1958). Il reste que le mouvement d'agitation culturelle et de traduction de la science-fiction américaine de l'immédiat après-guerre a suscité un lectorat ouvert aux valeurs de cette science-fiction, quand les auteurs français - et c'est là l'un des drames permanents de ces auteurs, à deux ou trois exceptions près - sont restés prisonniers de modèles discursifs qui ont plus à voir avec les récits de la science-fiction pré-moderne.

Nous n'entrerons pas ici dans l'analyse des positions assumées par les critiques de science-fiction, pour ne pas sortir de notre sujet, d'autant que le fait est patent: ce genre littéraire est vite ghettö̈sé dans un champ subculturel autonome en France comme il l'a été aux États-Unis. Les condamnations dont il a fait l'objet de la part des intellectuels et des journalistes de divers horizons n'ont d'autre effet sur son implantation que de conforter ses zélateurs dans la nécessité de constituer la science-fiction en un sous-champ autonome.

\section{Conclusion}

La traduction de la science-fiction américaine dans la France des années 1950 pose de façon aiguë des questions fondamentales à la traductologie. S'il est vrai que la traduction ne paraît connaître que des textes dans l'instant de sa réalisation, le texte risque d'être l'arbre qui cache la forêt de tous les autres textes que la dominance idéologique dans l'espace culturel regroupe en des types auxquels est consentie une niche socio-institutionnelle. La lutte pour le pouvoir symbolique ne passe jamais exclusivement par des textes saisis individuellement, mais par des classes et des types régis par les intérêts des groupes sociaux qui prennent position dans le champ. Dès lors, la question principale qui se pose, et qui concerne la mesure et la nature des transformations et des manipulations que subissent les textes dans le processus de la traduction, est la suivante. Lorsqu'un type de texte (ou un genre) prend corps dans un groupe social d'un espace culturel (source) et qu'il est traduit dans un autre espace culturel, par quel groupe social ce type de texte ou ce genre est-il reçu dans l'espace culturel cible? Dans le cas de la science-fiction américaine, le parachutage dans la France des années 1950 est une translation (au sens mathématique du terme) qui réussit du fait que le genre trouve en France un groupe que l'on peut tenir 146 
pour l'homologue de la petite bourgeoisie technophile qui aspire aux États-Unis à accroître son poids social. Boris Vian et Raymond Queneau en se faisant les agents de pointe de ce groupe ont largement contribué au succès de la naturalisation du genre grâce à la légitimité considérable qui est la leur dans le champ de la culture française de l'ćpoque.

Le sociologue de la science-fiction Gérard Klein rattache l'émergence de la science-fiction à la montée aux États-Unis d'une petite bourgeoisie à vocation technicienne (1977, p. 27), ce que corrobore à sa façon l'anthropologue culturel Leon Stover; pour Stover (1972), l'émergence et le succès de la science-fiction comme genre correspondent à l'industrialisation de la recherche aux États-Unis dans les années 1920, ce que l'on appelle communément Research and Development (qui n'est rien d'autre que la recherche appliquée, par opposition à la recherche fondamentale). La science-fiction avec son optimisme scientifique et technologique, son esprit de la nouvelle frontière (la conquête spatiale), son évocation d'altérités extraterrestres, théatralise un monde où le pouvoir est détenu par ceux qui n'ont pas peur du changement et de la métamorphose.

La traduction de la science-fiction comme genre américain est le symptôme littéraire d'un appel au changement de société. Mais les difficultés d'implantation et de reconnaissance, qui s'expriment globalement par la ghettoïsation et la marginalisation subculturelle, sont réelles. Elles sont à mettre sur le compte d'une disposition profondément ancrée dans l'habitus de la bourgeoisie française: la peur panique de la science et de la technologie en ce qu'elles sont senties confusément comme productrices de mutations sociales qui risquent de renverser les rapports de force dans la société.

La science-fiction américaine traduite jouit d'une position hégémonique depuis les années 1950 dans le champ de la science-fiction française. Près d'un demi-siècle après la constitution du champ de la science-fiction, rares sont les auteurs français qui se sont imposés à hauteur du succès de réception des auteurs américains traduits. En raison de l'immense capital symbolique dont ces derniers sont investis, les amateurs de ce genre en France boudent la plupart des auteurs français. Cette 
constatation oblig : à s'interroger sur la nature et la pérennité du capital symbolique des États-Unis dans la société française. Une partie de la réponse se trouve peut-être dans certains ouvrages publiés après la Seconde Guerre mondiale, par exemple le Défi américain de Jean-Jacques Servan-Schreiber publié en 1967. La question poséc peut se résumer ainsi: «Existe-t-il un avenir hors du modèle américain?» La science-fiction américaine des années 1920 à 1960 (jusqu'à grosso modo la «Spéculative-Fiction» d'Harlan Ellison également en $1967^{32}$ ) peut dans son ensemble être vue comme une thématisation de la prétention à l'universalité du modèle américain. La traduction devient alors une courroie de transmission dans l'entreprise de propagation du nationalisme des États-Unis ${ }^{33}$. Telle est sans doute la fonction majeure de la traduction de la science-fiction américaine en France. Cette fonction est située bien en deça des visées qui furent celles de Vian et de Queneau à la fin des années 1940 lorsqu'ils se firent les agents des valeurs de changement social telles qu'elles

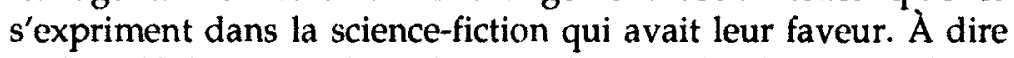
vrai, ce déplacement des enjeux est dans l'ordre des choses: le jeu social dans les champs du pouvoir s'inscrit dans l'espace négocié entre le possible social et la structure du champ (ici de la sciencefiction) tel qu'il s'est constitué par importation-traduction. La traduction $n^{\prime}$ est certes pas une simple translation (au sens

32. Harlan Ellison fait trembler les fondations de la science-fiction issue de Gernsback et J.W. Campbell Jr. en publiant Dangerous Visions, recueil de textes où le cadre des sciences pures et dures apparaît tout à coup trop étroit pour la nouvelle génération d'écrivains américains, qui préfèrent parler de «speculative fiction» au lieu de «science fiction».

33. Mais la traduction ne pourrait jouer ce rôle de courroie de transmission, s'il n'existait pas dans de larges pans de l'espace social français de l'après-gucre (pour ne pas préjuger de l'entre-deux-guerres) une adhésion inconsciente à l'American Way of Life comme la meilleure possible, adhésion fondée sur la conviction que la société américaine est nécessairement située à l'horizon de la société française. Cette disposition française à l'endroit de l'«Amérique» (revisitée à la française) fait de cette dernière un des joueurs sociaux majeurs, et ce quelles que soient les invectives dont elle a pu (et continue de) faire l'objet. 
mathématique ou même religieux du terme) d'un espace social à un autre espace social, puisque tout est susceptible d'être renégocié dans et par les luttes de pouvoirs de l'espace social cible. Mais c'est malgré tout une translation lorsque, comme dans la SF du ghetto subculturel, on constate le maintien de la dominance écrasante des modèles importés américains indépendamment des transformations subies par ces modèles.

\section{Université Concordia}

\section{Références}

\section{A. Le champ de la SF dans la France des années 1950}

\section{Les textes traduits par Boris Vian}

Frank M. Robinson, "Le Labyrinthe", Les Temps Modernes, $\mathrm{n}^{\circ} 72$, octobre 1951, pp. 628-646.

Wallace West, «les Vivisculpteurs», France-Dimanche, $\mathrm{n}^{\circ} 286,1952$, pp. 12-13.

Ray Bradbury, «le Veldt dans la nursery», France-Dimanche, $\mathrm{n}^{\circ}$ 288, 1952, p. 6.

William Tenn, "Pas bêtes, les gars de Bételgeuse», FranceDimanche, $\mathrm{n}^{\circ} 289,1952$, p. 10.

Murray Leinster, "Si vous étiez un Moklin", France-Dimanche, $\mathrm{n}^{\circ}$ 292, 1952, p. 9.

Lewis Padgett Ipseudonyme collectif de Henry Kuttner et Catherine Moorel: «Tout smouales étaient les borogoves», Mercure de France, $\mathbf{1}^{\text {er }}$ juin 1953.

Alfred E. Van Vogt, Le Monde des $\bar{A}$. Paris, Gallimard, «Le Rayon Fantastique», 1953.

Alfred E. Van Vogt, Les Aventures de $\bar{A}$. Paris, Gallimard, "Le Rayon Fantastique», 1957. 


\section{Les articles sur la SF par Vian, Spriel, Queneau}

QUENEAU, Raymond. «Un nouveau genre littéraire: les Science fictions", Critique, $\mathrm{n}^{\circ} 16$, mars 1951.

novembre 1953.

. «La science-fiction vaincra», Arts, 29 octobre-4

VIAN, Boris et Stéphane [sic] Spriel. «Un nouveau genre littéraire: la Science-fiction", Les Temps modernes, $\mathrm{n}^{\circ} 72$, octobre 1951, pp. 618-627.

. «Un robot-poète ne nous fait pas peur», Arts, 10-16 avril 1953. Repris dans Cantilènes en geléc, Paris, U.G.E., 1970.

. «Sur certains aspects actuels de la science-fiction", $L a$ Parisienne, $\mathrm{n}^{\circ} 11$, novembre 1953, pp. 1541-1544.

Entretien: «Pierre Kast et Boris Vian s'entretiennent de la Sciencefiction», L'Écran, n 1, janvier 1958.

\section{B. Références secondaires}

\section{Ouvrages de théorie et $d^{\prime}$ histoire de la science-fiction}

ANGENOT, Marc (1980). «La science-fiction: genre et statut institutionnel», Reoue de l'Institut de sociologie, $\mathrm{n}^{\circ} 3 / 4, \mathrm{pp} .651-660$.

GOUANVIC, Jean-Marc (1981). «Positions de l'histoire dans la science-fiction", Cahiers du collectif Change, $n^{\circ}$ 40, mars, pp. 85-103.

KETTERER, David (1974). New Worlds for Old. The Apocalyptic Imagination, Science Fiction, and American Literature. New York, Anchor Books.

KLEIN, Gérard (1977). Malaise dans la science-fiction. Metz, L'Aube enclavée.

STOVER, Leon (1972). La Science-fiction américaine - Essai d'anthropologie culturelle. Paris, Aubier-Montaigne. 
SUVIN, Darko (1979). Metamorphoses of Science Fiction. On the Poetics and History of a Literary Genre. New Haven and London, Yale University Press.

\section{La sociologie des biens symboliques}

BOURDIEU, Pierre (1971). «Le marché des biens symboliques», L'Année sociologique, vol. 22, pp. 49-126.

Paris, Minuit, coll. «Le sens commun».

(1979). La Distinction - Critique sociale du jugement.

(1982). Leçon sur la leçon. Paris, Minuit. [Leçon inaugurale prononcée au Collège de France le vendredi 23 avril 1982.]

commun».

(1987). Choses dites. Paris, Minuit, coll. «Le sens

(1992). Les Règles de l'art. Cenèse et structure du champ littéraire. Paris, Seuil, coll. «Libre examen».

RÉSUMÉ: La traduction et le devenir social: le cas de l'irruption de la science-fiction américaine en France après la Seconde Guerre mondiale. À partir de la théorie de Pierre Bourdieu (concepts de champ, de capital et de biens symboliques, d'habitus ct d'illusio), cette étude propose une sociologie de la traduction appliquée à l'importation de la science-fiction américaine en France dans les années 1950. C'est d'un "nouveau genre littéraire" d'origine américaine que Boris Vian, Raymond Queneau et Michel Pilotin se font les initiateurs dans l'espace socio-culturel français. Or, si les textes de SF des auteurs américains sont traduits massivement dans la culture française de l'époque, cette traduction n'a lieu que moyennant l'importation des structures institutionnelles américaines autonomes (en particulier des magazines et des collections spécialisées) qui ont émergées à la fin des années 1920 et à la naturalisation du modèle subculturel américain qui aboutissent à la constitution d'un champ de science-fiction autonome dans l'espace culturel français. Dès lors, la question traductologique essentielle que pose l'importation de la science-fiction américaine en France est la suivante. Lorsqu' un type de texte (ou un genre) prend corps dans un groupe social d'un espace cul! sel (source) ct qu'il est traduit dans un 
autre espace culturel, par quel groupe social ce type de texte ou ce genre est-il reçu dans l'espace culturel cible? L'auteur fait l'hypothèse que la translation (au sens mathématique du terme) de la science-fiction américaine (textes et structures institutionnelles) réussit parce que, d'une part, il existe en France une ou des catégories sociales qui sont les homologues de la petite bourgeoisie américaine technophile des années 1920 et parce que, d'autre part, il existe une adhésion plus ou moins consciente à l'American way of life comme le modèle de société qui s'impose comme allant de soi dans de larges pans de l'espace social français de l'après-guerre. Dans ces conditions, la traduction contribue à renforcer le modèle américain dans sa prétention à l'universalité, quelles qu'aient été à l'origine les vertus de changement social que les agents d'implantation Vian, Queneau et Pilotin avaient reconnues dans la science-fiction et sur lesquelles ils s'appuyaient pour la présenter comme un «genre nouveau».

ABSTRACT: Translation and the "Social Future": the Irruption of American Science Fiction in France after the Second World War Using the theory of Pierre Bourdieu (the concepts of field, of capital and symbolic goods, of habitus, and illusio), this article provides a study in the sociology of translation applied to the importing of American science fiction into France during the 1950's. Boris Vian, Raymond Queneau and Michel Pilotin are the promoters of an alleged "new literary genre" within the French socio-cultural context. But the massive translation of American SF authors during this period was possible only with the creation of imported institutional structures, in particular specialized magazines and book series, which emerged in the U.S. at the end of the 1920 s, and with the naturalization of the American subcultural model which had as its result the constitution of an autonomous field of science fiction in France. The crucial question for translation studies is the following: When a new text-type or genre is incorporated into a new cultural context, which social group(s) receive(s) this text-type or genre within the target culture, and according to what conditions? The author suggests that the "translation" (in the mathematical sense) of American science fiction (texts and institutional structures) was successful because, on the one hand, there existed in France social categories homologous to the technophile American middle class of the 1920 s and, on the other hand, because there was a more or less conscious adherence to the American way of life as a social model in large sectors of post-war French society. Translation then contributed to strengthening the American pretension to universality, even though Vian, Queneau and Pilotin had sought rather to exploit the high potential for social change they had recognized in the "new genre" of science fiction. 